\title{
Constructing the CKVs of Bianchi III and V spacetimes
}

\author{
Antonios Mitsopoulos ${ }^{1 *}$, Michael Tsamparlis ${ }^{1 \dagger}$ and Andronikos Paliathanasis ${ }^{2 \ddagger}$ \\ ${ }^{1}$ Faculty of Physics, Department of Astronomy-Astrophysics-Mechanics, \\ University of Athens, Panepistemiopolis, Athens 157 83, Greece. \\ ${ }^{2}$ Institute of Systems Science, Durban University of Technology \\ Durban 4000, Republic of South Africa.
}

\begin{abstract}
We determine the conformal algebra of Bianchi III and Bianchi V spacetimes or, equivalently, we determine all Bianchi III and Bianchi V spacetimes which admit a proper conformal Killing vector. The algorithm that we use has been developed in Class. Quantum. Grav. 15, 2909 (1998) and concerns the computation of the CKVs of decomposable spacetimes. The main point of this method is that a decomposable space admits a CKV if the reduced space admits a gradient homothetic vector the latter being possible only if the reduced space is flat or a space of constant curvature. We apply this method in a stepwise manner starting from the two dimensional spacetime which admits an infinite number of CKVs and we construct step by step the Bianchi III and V spacetimes by assuming that CKVs survive as we increase the dimension of the space. We find that there is only one Bianchi III and one Bianchi V spacetime which admit at maximum one proper $\mathrm{CKV}$. In each case we determine the conformal Killing vector and the corresponding conformal factor. As a first application in these two spacetimes we study the kinematics of the comoving observers and the dynamics of the corresponding cosmological fluid. As a second application we determine in these spacetimes generators of the Lie symmetries of the wave equation.

Keywords: Bianchi spacetimes; Conformal vector fields; Collineations; Symmetries; Lie symmetries of wave equation.
\end{abstract}

\section{Introduction}

A conformal Killing vector (CKV) $\mathbf{X}$ of a metric $g_{a b}$ is a vector field that satisfies the condition $L_{\mathbf{X}} g_{a b}=2 \psi g_{a b}$ where $\psi\left(x^{r}\right)$ is the conformal factor. The CKVs are classified as Killing Vectors (KVs) for $\psi=0$; Homothetic Vectors (HVs) for $\psi=$ const; Special Conformal Killing Vectors (SCKVs) for $\psi_{; a b}=0$; and proper CKVs for $\psi_{; a b} \neq 0$. The HVs and the KVs are also non-proper Affine Collineations and non-proper Projective Collineations.

The knowledge of the proper CKVs of a given spacetime is important because they act as geometric constraints which can be used in the study of the kinematics and the dynamics of a given spacetime. For example a CKV can be used to reduce the number of unknowns of a gravitational (or cosmological) model and also to increase the possibility of finding new solutions of Einstein's field equations (see for example [1], [2, [3], 4], [5], 6], 7], 8, 9]). Furthermore the conformal algebra can be used in order to classify spaces (e.g. Finsler manifolds, pseudo-Euclidean manifolds) (see [10, 11]). For example one may use the CKVs of a space in order to determine the classes of manifolds which are conformally related to the given space; or to use them in order to study the locally conformal flatness of a space around a singularity (i.e. a point $x_{0}$ where the CKV vanishes). The special class of the CKVs, the KVs, have been used in numerous applications. For example Geroch in [12] and 13 has used the KVs in order to derive new solutions of the gravitational field equations. The gradient KVs can be used also to decompose the spacetime metric and simplify the field equations. Moreover, KVs are

\footnotetext{
*Email:antmits@phys.uoa.gr

$\dagger$ Email: mtsampa@phys.uoa.gr

‡Email: anpaliat@phys.uoa.gr
} 
related with the conservation laws for the geodesic equations, indeed KVs form a subalgebra on the Noether symmetries for the geodesic Lagrangian, for more details see [14]

Apart of the above applications, another important area where the CKVs and the more general projective collineations have been used is the geometric study of Lie symmetries of differential equations. For example early studies of the geodesic equations [15], [16], [17, [18] have shown a unique connection of the Lie point symmetries for the geodesic equations in a space with the elements of the projective algebra of the space. Furthermore in a conservative dynamical system one may consider the kinetic energy as a metric and study the Lie and the Noether point symmetries of the dynamic equations using the collineations of this metric. In [19, 20] it has been shown that in these case the Lie point symmetries are generated by the special projective algebra and the Noether point symmetries by the Homothetic algebra of the kinetic metric. Similar results have been found for some partial differential equations of special interest in curved spacetimes, as the wave and the heat equation (see [21], 22], 23] and references therein) where it has been shown that in these cases the Lie point symmetries involve the CKVs.

In the present work we apply the propositions and the methodology developed in [24, 25] and 28] in order to determine all Bianchi III and V spacetimes that admit proper Conformal Killing Vectors (CKVs). The Bianchi I spacetimes which admit proper CKVs have been determined in [28. Bianchi III and Bianchi V spacetimes are of special interest and they find many applications in the study of anisotropic cosmologies. Bianchi can provide a different cosmological behaviour in the early universe [26, while also they can been seen as the homogeneous limits of exact inhomogeneous cosmological models [27].

Bianchi spacetimes are spatially homogeneous spacetimes of the general form

$$
d s^{2}=-d t^{2}+A^{2}(t)\left(\omega_{1}\right)^{2}+B^{2}(t)\left(\omega_{2}\right)^{2}+C^{2}(t)\left(\omega_{3}\right)^{2}
$$

where $\omega_{i}, i=1,2,3$, are basis 1 -forms and $A(t), B(t), C(t)$ are functions of the time coordinate (see 29], 30], [31]). For instance,

$$
\begin{aligned}
& \text { Bianchi I : } \omega_{1}=d x, \omega_{2}=d y, \omega_{3}=d z \\
& \text { Bianchi III : } \omega_{1}=d x, \omega_{2}=d y, \omega_{3}=e^{-x} d z \\
& \text { Bianchi V : } \omega_{1}=d x, \omega_{2}=e^{x} d y, \omega_{3}=e^{x} d z \text {. }
\end{aligned}
$$

In the case of $B^{2}(t)=C^{2}(t)$ the Bianchi spacetimes contain a fourth isometry which is the rotation of the $y z$ plane and reduce to the important subclass of Locally Rotational Symmetric (LRS) spacetimes (see for example 32 and citations therein).

The structure of the paper is as follows. In section 2 we briefly discuss the method we apply for the determination of the proper CKVs of a decomposable spacetime. Sections 3 and 4 contain the main calculations of the paper and the propositions derived from the application of the method mentioned in section 2. In section [5 we apply our results in the cases of Bianchi III and Bianchi V cosmological models. Section [6 contains an application of the CKVs on the symmetries of differential equations. More specifically, we compute the generic Lie point symmetries of the wave equation for the Bianchi spacetimes constructed in sections 3 and 4 Finally, in section 7 we draw our conclusions.

\section{Preliminaries}

We recall that a Riemannian manifold is decomposable along the coordinate $t$ iff the metric $g_{a b}$ admits the non-null gradient KV $u^{a}=\partial t$ where $u^{a} u_{a}=\varepsilon u^{2}, \varepsilon= \pm 1$. In this case one defines the projection operator

$$
h_{a b}=g_{a b}-\frac{\varepsilon}{u^{2}} u_{a} u_{b}
$$

and decomposes the tensor algebra along $u^{a}$ and normal to $u^{a}$. For $n$-dimensional decomposable Riemannian manifolds $M^{n}$ with $n \geq 3$ an algorithm has been developed (see 24]) which determines the proper CKVs in terms of the (gradient) proper CKVs of the $(n-1)$ non-decomposable space.

In particular, it has been shown that an $n$-dimensional decomposable space $M^{n}$ admits proper CKVs if and only if the $(n-1)$ space $M^{n-1}$ admits a gradient proper CKV whose conformal factor is the gradient factor which constructs the (gradient) CKV. In addition, any gradient proper CKV of the $M^{n-1}$ provides two proper CKVs for the $M^{n}$. For a four dimensional manifold the following result is shown in [24]. 
If $M^{n}, n=4$ is a decomposable Riemannian manifold with line element $(\mu, \nu=1,2,3)$

$$
d s^{2}=\varepsilon d t^{2}+h_{\mu \nu}\left(x^{\sigma}\right) d x^{\mu} d x^{\nu}
$$

where $\varepsilon= \pm 1$, the vector field

$$
X^{a} \partial_{a}=-\frac{\varepsilon}{p} \dot{\lambda}(t) \psi\left(x^{\sigma}\right) \partial_{t}+\frac{1}{p} \lambda(t) \xi^{\mu}\left(x^{\sigma}\right) \partial_{\mu}+L^{\mu} \partial_{\mu}
$$

is a proper CKV of (3) where

a. $L^{\mu}$ is a non-gradient $\mathrm{KV}$ or $\mathrm{HV}$ of $M^{n-1}$

b. $\xi^{\mu}\left(x^{\sigma}\right)$ is a gradient proper CKV of $M^{n-1}$ with conformal factor $\psi\left(x^{\sigma}\right)$ i.e. $L_{\xi} h_{\mu \nu}\left(x^{\sigma}\right)=2 \psi\left(x^{\sigma}\right) h_{\mu \nu}\left(x^{\sigma}\right)$

c. The function $\lambda(t)$ is given by

$$
\lambda(t)=\lambda_{1} e^{i \sqrt{\varepsilon p} t}+\lambda_{2} e^{-i \sqrt{\varepsilon p} t}, \text { for } \varepsilon p>0
$$

or

$$
\lambda(t)=\lambda_{1} e^{\sqrt{-\varepsilon p} t}+\lambda_{2} e^{-\sqrt{-\varepsilon p} t}, \text { for } \varepsilon p<0
$$

where $p$ is a non-vanishing constant and $\lambda_{1}, \lambda_{2}$ are independent constants, provided the function $\psi\left(x^{\sigma}\right)$ satisfies the condition

$$
\psi_{; \mu \nu}=p \psi h_{\mu \nu} .
$$

Concerning the homothetic vector it has been shown in 24] that when the $M^{n-1}$ space admits a HV $H^{\mu}\left(x^{\sigma}\right)$ with conformal factor $C$, the $M^{n}$ admits the $\mathrm{HV}$

$$
H^{a} \partial_{a}=C t \partial_{t}+H^{\mu} \partial_{\mu}
$$

Finally, concerning the Killing vector fields, it has been shown that the Killing vector fields of $M^{n}$ are

$$
K^{a}=k_{0} \partial_{t}+k_{1 I} h^{\mu \nu}\left(x^{\sigma}\right) K_{\nu}^{I}\left(x^{\sigma}\right)+k_{2 I} h^{\mu \nu}\left(x^{\sigma}\right) S_{, \nu}^{I}\left(x^{\sigma}\right)+k_{3 I}\left(-\varepsilon S^{I}\left(x^{\sigma}\right) \partial_{t}+h^{\mu \nu}\left(x^{\sigma}\right) S_{, \nu}^{I}\left(x^{\sigma}\right)\right)
$$

where $K_{\nu}^{I}\left(x^{\sigma}\right)$ are the non-gradient KVs of $M^{n-1}$ and $S_{, \nu}^{I}\left(x^{\sigma}\right)$ are the gradient KVs of $M^{n-1}$. Finally, $k_{0}, k_{1 I}$, $k_{2 I}$ and $k_{3 I}$ are independent constants.

However another possibility that the space (3) admits proper CKVs is when it is conformally flat. That case was found to be important in the classification of Bianchi I spacetimes in 28. according to the admitted CKVs, but it does not provide any result in the case of Bianchi III and Bianchi V spacetimes, thus we omit it from the present discussion.

The concept of conformally related metrics plays a crucial role in the computation of the CKVs, therefore we review the basic definitions concerning these metrics. Two metrics $\widehat{g}_{a b}, g_{a b}$ are said to be conformally related iff there is a function $N^{2}\left(x^{r}\right)$ such that $\widehat{g}_{a b}=N^{2}\left(x^{r}\right) g_{a b}$. The conformally related metrics share the same conformal algebra but with different conformal factors. For a given vector field $\mathbf{X}$ we have the decompositions/identities

$$
L_{\mathbf{X}} \widehat{g}_{a b}=2 \widehat{\psi}(\mathbf{X}) \widehat{g}_{a b}+2 \widehat{H}_{a b}(\mathbf{X}) \text { and } L_{\mathbf{X}} g_{a b}=2 \psi(\mathbf{X}) g_{a b}+2 H_{a b}(\mathbf{X}) .
$$

where $H_{a b}(\mathbf{X}), \widehat{H}_{a b}(\mathbf{X})$ are symmetric traceless tensors. It can be shown that

$$
\widehat{\psi}(\mathbf{X})=\mathbf{X}(\ln N)+\psi(\mathbf{X}), \quad \widehat{H}_{a b}(\mathbf{X})=N^{2} H_{a b}(\mathbf{X})
$$

and

$$
\widehat{F}_{a b}(\mathbf{X})=N^{2} F_{a b}(\mathbf{X})-2 N N_{,[a} X_{b]}
$$

where $\widehat{F}_{a b}(\mathbf{X})=\widehat{X}_{[a ; b]}=\widehat{X}_{[a ; b]}$ and $F_{a b}(\mathbf{X})=X_{[a ; b]}$. Moreover

$$
\begin{aligned}
\widehat{X}_{a ; b} & =\frac{1}{2} L_{\mathbf{X}} \widehat{g}_{a b}+\widehat{F}_{a b}(\mathbf{X}) \\
X_{a ; b} & =\frac{1}{2} L_{\mathbf{X}} g_{a b}+F_{a b}(\mathbf{X}) .
\end{aligned}
$$


A metric $g_{a b}$ is called conformally flat iff it is conformal to the flat metric $\eta_{a b}$. A metric conformally related to a conformally flat metric is also conformally flat. It is well known that all the $2 \mathrm{~d}$-spacetimes are conformally flat and admit an infinity number of CKVs while only the flat 2d-metrics admit special CKVs.

The flat $n$-dimensional metric $\eta_{a b}$ admits an algebra of $\frac{(n+1)(n+2)}{2}$ CKVs which consists of $n+\frac{n(n-1)}{2}=\frac{n(n+1)}{2}$ $\mathrm{KVs}$, one HV and $n$ proper SCKVs. The generic (not proper!) SCKV of a flat metric is given by the formula

$$
\chi^{a} \partial_{a}=\alpha^{a} \mathbf{P}_{a}+\alpha^{B A} \mathbf{r}_{A B}+\beta \mathbf{H}+2 \beta^{a} \mathbf{K}_{a}
$$

with conformal factor $\psi=\beta+2 \beta_{a} x^{a}$ and integration constants $\alpha, \beta, \alpha^{a}, \beta^{a}, \alpha_{a b}=-\alpha_{b a}$. In this expression $\mathbf{P}_{a}$ are the $n \mathrm{KVs}$ (translations), $\mathbf{r}_{A B}$ are the $\frac{n(n-1)}{2} \mathrm{KVs}$ (rotations), $\mathbf{H}$ is the $\mathrm{HV}$ (dilatation) and $\mathbf{K}_{a}$ are the $n$ proper SCKVs. The summation over $A, B$ satisfies the condition $1 \leq A<B \leq n$.

In a coordinate system in which the metric has its reduced form $\eta_{a b}=\operatorname{diag}(-1, \ldots,-1,+1, \ldots,+1)$ the above vectors are given by the following expressions $\mathbf{P}_{a}=\delta_{a}^{b} \partial_{b}, \mathbf{r}_{a b}=2 \delta_{[a}^{c} \delta_{b]}^{d} x_{c} \partial_{d}, \mathbf{H}=x^{a} \partial_{a}$ and $\mathbf{K}_{a}=$ $\left(x_{a} x^{b}-\frac{1}{2} \delta_{a}^{b} x_{c} x^{c}\right) \partial_{b}=x_{a} \mathbf{H}-\frac{1}{2}\left(x_{b} x^{b}\right) \mathbf{P}_{a}$. These vector fields span the conformal algebra of the flat space $\eta_{i j}$.

\section{CKVs of Bianchi III spacetime}

Consider the three-dimensional decomposable spacetime of Lorentzian signature

$$
d s_{(1+2)}^{2}=\Gamma^{2}(\tau)\left(-d \tau^{2}+d x^{2}\right)+d y^{2} .
$$

The line element (11) for arbitrary $\Gamma(\tau)$ admits a two-dimensional conformal Killing algebra consisting by the KVs $\partial_{y}$ and $\partial_{x}$.

For the conformal spacetime

$$
d \bar{s}_{(1+2)}^{2}=B^{2}(\tau) e^{2 x} d s_{(1+2)}^{2}
$$

the vector field $\partial_{y}$ remains a KV but $\partial_{x}$ now becomes a proper $\mathrm{HV}$.

Consider now the four-dimensional decomposable spacetime

$$
d s_{(1+3)}^{2}=d \bar{s}_{(1+2)}^{2}+d z^{2}
$$

which admits a three-dimensional conformal algebra consisting of the KVs $\partial_{y}, \partial_{z}$ and the proper $\mathrm{HV} \partial_{x}+z \partial_{z}$. Then, the conformally related spacetime $d s_{(I I I)}^{2}=A^{2}(\tau) e^{-2 x} d s_{(1+3)}^{2}$ which can be written equivalently 1

$$
d s_{(I I I)}^{2}=-d t^{2}+\alpha^{2}(t) d x^{2}+\beta^{2}(t) d y^{2}+\gamma^{2}(t) e^{-2 x} d z^{2}
$$

is a Bianchi III spacetime and the vector fields $\partial_{y}, \partial_{z}, \partial_{x}+z \partial_{z}$ form the Killing algebra of (14). Therefore, in order the Bianchi III (14) to admit greater conformal algebra the functions $\alpha(t), \beta(t)$ and $\gamma(t)$ must be specified. Recall that when $\alpha(t)=\gamma(t)$ spacetime (14) is locally rotational and admits as extra KV the rotation in the two dimensional space $d s^{2}=d x^{2}+e^{-2 x} d z^{2}$.

The three-dimensional space (11) admits a greater conformal algebra for specific functions $\Gamma(\tau)$. From the discussion of Section 2 it follows that $\Gamma(\tau)$ must be such that the two-dimensional space

$$
d s_{(2)}^{2}=\Gamma^{2}(\tau)\left(-d \tau^{2}+d x^{2}\right)
$$

admits proper gradient CKVs or a greater Killing algebra. For two-dimensional spaces it is well-known that the admitted KVs can be zero, one or three and in the latter case the space is maximally symmetric. Since (15) admits always the $\mathrm{KV} \partial_{x}$, the $\Gamma(\tau)$ must be specified so that (15) is maximally symmetric. Without loss of generality we can select $\Gamma^{2}(\tau)=e^{m \tau}$ in which case (15) is the flat space with Ricci Scalar $R_{(2)}=0$, or $\Gamma^{2}(\tau)=\kappa^{-2} \cos ^{-2}(\tau)$ in which case $R_{(2)}=2 \kappa^{2}$.

Furthermore, because all the two-dimensional spaces admit infinitely many CKVs, the requirement that at least one of the proper CKVs is to be gradient, specifies the spacetime to be of nonzero constant curvature, which is a maximally symmetric space and it admits five gradient proper CKVs.

\footnotetext{
${ }^{1}$ Where $\alpha^{2}(t)=A^{2}(t) B^{2}(t) \Gamma^{2}(t), \beta^{2}(t)=A^{2}(t) B^{2}(t)$ and $\gamma^{2}(t)=A^{2}(t)$ while $t=\int a(\tau) d \tau$.
} 


\subsection{Case $\Gamma^{2}(\tau)=e^{m \tau}$}

In the case of $\Gamma^{2}(\tau)=e^{m \tau}$ the three-dimensional space

$$
d s_{(1+2)}^{2}=e^{m \tau}\left(-d \tau^{2}+d x^{2}\right)+d y^{2} .
$$

is flat and admits a ten-dimensional conformal algebra. This algebra consists of the six KVs

$$
\begin{gathered}
\mathbf{Y}_{1}=\frac{2}{m} e^{-\frac{m}{2}(\tau-x)} \partial_{\tau}-\frac{2}{m} e^{-\frac{m}{2}(\tau-x)} \partial_{x} \\
\mathbf{Y}_{2}=-\frac{2}{m} e^{-\frac{m}{2}(\tau+x)} \partial_{\tau}-\frac{2}{m} e^{-\frac{m}{2}(\tau+x)} \partial_{x} \\
\mathbf{Y}_{3}=\partial_{x}, \quad \mathbf{Y}_{4}=\partial_{y} \\
\mathbf{Y}_{5}=y e^{-\frac{m}{2}(\tau+x)} \partial_{\tau}+y e^{-\frac{m}{2}(\tau+x)} \partial_{x}+\frac{2}{m} e^{\frac{m}{2}(\tau-x)} \partial_{y} \\
\mathbf{Y}_{6}=-y e^{-\frac{m}{2}(\tau-x)} \partial_{\tau}+y e^{-\frac{m}{2}(\tau-x)} \partial_{x}-\frac{2}{m} e^{\frac{m}{2}(\tau+x)} \partial_{y}
\end{gathered}
$$

the HV

$$
\mathbf{Y}_{7}=\frac{2}{m} \partial_{\tau}+y \partial_{y}, \psi_{(1+2)}\left(\mathbf{Y}_{7}\right)=1
$$

and the three special CKVs

$$
\begin{gathered}
\mathbf{Y}_{8}=\left[\frac{2}{m^{2}} e^{\frac{m}{2}(\tau-x)}+\frac{y^{2}}{2} e^{-\frac{m}{2}(\tau+x)}\right] \partial_{\tau}+\left[-\frac{2}{m^{2}} e^{\frac{m}{2}(\tau-x)}+\frac{y^{2}}{2} e^{-\frac{m}{2}(\tau+x)}\right] \partial_{x}+\frac{2 y}{m} e^{\frac{m}{2}(\tau-x)} \\
\mathbf{Y}_{9}=-\left[\frac{2}{m^{2}} e^{\frac{m}{2}(\tau+x)}+\frac{y^{2}}{2} e^{-\frac{m}{2}(\tau-x)}\right] \partial_{\tau}+\left[-\frac{2}{m^{2}} e^{\frac{m}{2}(\tau+x)}+\frac{y^{2}}{2} e^{-\frac{m}{2}(\tau-x)}\right] \partial_{x}-\frac{2 y}{m} e^{\frac{m}{2}(\tau+x)} \\
\mathbf{Y}_{10}=m y \partial_{\tau}+\left[\frac{m^{2} y^{2}}{4}+e^{m \tau}\right] \partial_{y}
\end{gathered}
$$

with conformal factors $\psi_{(1+2)}\left(\mathbf{Y}_{8}\right)=\frac{2}{m} e^{\frac{m}{2}(\tau-x)}, \psi_{(1+2)}\left(\mathbf{Y}_{9}\right)=-\frac{2}{m} e^{\frac{m}{2}(\tau+x)}$, and $\psi_{(1+2)}\left(\mathbf{Y}_{10}\right)=\frac{m^{2} y}{2}$ respectively.

The conformally flat space

$$
d \bar{s}_{(1+2)}^{2}=B^{2}(\tau) e^{2 x}\left[e^{m \tau}\left(-d \tau^{2}+d x^{2}\right)+d y^{2}\right]
$$

admits the same elements of the conformal algebra with (16) but with different conformal factors $\bar{\psi}_{(1+2)}$. More specifically it follows that

$$
\bar{\psi}_{(1+2)}\left(\mathbf{Y}_{A}\right)=\mathbf{Y}_{A}\left[\ln \left(B e^{x}\right)\right]+\psi_{(1+2)}\left(\mathbf{Y}_{A}\right) .
$$

When we impose condition (77) we find that there does not exist function $B(\tau)$ such that $\bar{\psi}_{(1+2)}\left(\mathbf{Y}_{A}\right)$ to satisfy (17). On the other hand, we observe that for

$$
B(\tau)=e^{\mu \tau}, \quad \mu=\frac{m(\lambda-1)}{2}
$$

it follows $\bar{\psi}_{(1+2)}\left(\mathbf{Y}_{7}\right)=\lambda$, which means that $Y_{7}$ is reduced to a HV for (17). At this point it is important to mention that $\bar{\psi}_{1+2}\left(\mathbf{Y}_{3}\right)=1$, however there is only one proper $\mathrm{HV}$ and not two, as expected. We assume $\mathbf{Y}_{7}$ to be the proper $\mathrm{HV}$ and $\mathbf{Y}_{3}-\frac{1}{\lambda} \mathbf{Y}_{7}$ to be a $\mathrm{KV}$.

For the four-dimensional decomposable spacetime

$$
d s_{(1+3)}^{2}=e^{2 x} e^{2 \mu \tau}\left[e^{m \tau}\left(-d \tau^{2}+d x^{2}\right)+d y^{2}\right]+d z^{2}
$$

from $Y_{7}$ we find the proper $\mathrm{HV}$

$$
\mathbf{L}_{1} \equiv \mathbf{Y}_{7}+\lambda z \partial_{z}=\frac{2}{m} \partial_{\tau}+y \partial_{y}+\lambda z \partial_{z}
$$


We conclude that the Bianchi III spacetime

$$
d s_{(I I I)}^{2}=e^{m \lambda \tau} A^{2}(\tau)\left(-d \tau^{2}+d x^{2}+e^{-m \tau} d y^{2}+e^{-m \lambda \tau} e^{-2 x} d z^{2}\right)
$$

admits the proper CKV $\mathbf{L}_{1}$ with conformal factor $\psi_{(I I I)}\left(\mathbf{L}_{1}\right)=\frac{2}{m} \frac{A_{,} \tau}{A}+\lambda$ which reduces to a $\mathrm{HV}$ when $A(\tau)$ is an exponential in which case the line element is

$$
d s_{(I I I)}^{2}=-e^{m \kappa \tau} d \tau^{2}+e^{m \kappa \tau} d x^{2}+e^{m(\kappa-1) \tau} d y^{2}+e^{m(\kappa-\lambda) \tau} e^{-2 x} d z^{2}
$$

or in equivalent form

$$
d s_{(I I I)}^{2}=-d t^{2}+\frac{m^{2} \kappa^{2} t^{2}}{4} d x^{2}+\left(\frac{m^{2} \kappa^{2} t^{2}}{4}\right)^{\frac{\kappa-1}{\kappa}} d y^{2}+\left(\frac{m^{2} \kappa^{2} t^{2}}{4}\right)^{\frac{\kappa-\lambda}{\kappa}} e^{-2 x} d z^{2}
$$

where now we write $\mathbf{L}_{1}=\kappa t \partial_{t}+y \partial_{y}+\lambda z \partial_{z}$ with $\psi_{(I I I)}\left(\mathbf{L}_{1}\right)=$ const $\equiv \kappa \neq 0$, recall that $d t=e^{\frac{m \kappa}{2} \tau} d \tau$.

Performing the same analysis for the second case of $\Gamma^{2}(\tau)=\kappa^{-2} \cos ^{-2}(\tau)$ we find that the resulting Bianchi III spacetime does not admit any proper CKV or a proper HV, hence we omit the presentation of this analysis.

We summarize our results in the following proposition

Proposition 1 The only Bianchi III spacetime which admits a proper CKV is the

$$
d s^{2}=A^{2}(\tau)\left[e^{m \lambda \tau}\left(-d \tau^{2}+d x^{2}\right)+e^{m(\lambda-1) \tau} d y^{2}+e^{-2 x} d z^{2}\right] .
$$

The $C K V$ is $\mathbf{L}_{1}=\frac{2}{m} \partial_{\tau}+y \partial_{y}+\lambda z \partial_{z}$ and it has conformal factor $\psi_{(I I I)}\left(\mathbf{L}_{1}\right)=\frac{2}{m} \frac{A_{, \tau}}{A}+\lambda$, where $A(\tau)$ is an arbitrary function.

\section{Bianchi V spacetimes which admit a CKV}

For the computation of the CKVs for the Bianchi V spacetime we apply the same procedure with Section 3 , but for this case we start from the two-dimensional spacetime

$$
d s_{(2)}^{2}=\Gamma^{2}(\tau) e^{-2 x}\left(-d \tau^{2}+d x^{2}\right) .
$$

The latter space is maximally symmetric only for $\Gamma^{2}(\tau)=e^{\gamma \tau}$ where the Ricci Scalar is calculated to be $R_{(2)}=0$. It is important to mention that there does not exist a function $\Gamma(\tau)$ such that the space (26) is of constant curvature.

We omit the intermediary calculations and we summarize the results in the following proposition

Proposition 2 The Bianchi V spacetime

$$
d s^{2}=A^{2}(\tau)\left[\Gamma^{2}(\tau)\left(-d \tau^{2}+d x^{2}\right)+e^{2 x}\left(B^{2}(\tau) d y^{2}+d z^{2}\right)\right]
$$

admits the unique proper $C K V \mathbf{L}_{1}=\frac{2}{m} \partial_{\tau}+y \partial_{y}+\lambda z \partial_{z}$ with $\psi_{(V)}\left(\mathbf{L}_{1}\right)=\frac{2}{m} \frac{A_{, \tau}}{A}+\lambda$ only when $\Gamma^{2}(\tau)=$ $e^{m \lambda \tau}, B^{2}(\tau)=e^{m(\lambda-1) \tau}$. For $A^{2}(\tau)=e^{m(\kappa-\lambda) \tau}$ the $C K V$ reduces to a $H V$ with conformal factor $\psi_{(V)}\left(\mathbf{L}_{1}\right)=$ const $=\kappa \neq 0$.

\section{Applications}

\subsection{Bianchi III cosmological fluid}

In this section we study some of the kinematic and the dynamic properties of spacetime given by equation (22) for the comoving observers $u^{a}=\frac{e^{-\frac{m \lambda}{2} \tau}}{A(\tau)} \delta_{\tau}^{a}, u^{a} u_{a}=-1$. As it is well known (see e.g. [33]) the four velocity of a class of observers introduces the $1+3$ decomposition of tensor fields in spacetime. The decomposition of $u_{a ; b}$ gives the kinematic quantities $\theta, \sigma^{2}, \omega^{2}$ and $\alpha^{a}$ defined by the identity

$$
u_{a ; b}=-\alpha_{a} u_{b}+\omega_{a b}+\sigma_{a b}+\frac{1}{3} \theta h_{a b}
$$


where $a^{a}=\dot{u}^{a}=u_{; b}^{a} u^{b}, \omega_{a b}=h_{a}^{c} h_{b}^{d} u_{[c ; d]}, \sigma_{a b}=\left(h_{a}^{c} h_{b}^{d}-\frac{1}{3} h^{c d} h_{a b}\right) u_{(c ; d)}, \theta=h^{a b} u_{a ; b}=u_{; a}^{a}, \sigma^{2} \equiv \frac{1}{2} \sigma_{a b} \sigma^{a b}$, $\omega^{2} \equiv \frac{1}{2} \omega_{a b} \omega^{a b}, h_{a b}=g_{a b}+u_{a} u_{b}$. In this decomposition $a^{a}$ is the 4-acceleration of the observers $u^{a}$, and the quantities $\omega_{a b}, \sigma_{a b}, \theta$ concerns the variation of the projected $\left(\perp \delta x^{a} u_{a}=0\right)$ connecting vector $\perp \delta x^{a}$ along the congruence (i.e. the integral lines) defined by the vector field $u^{a}$. The antisymmetric tensor $\omega_{a b}$ measures the relative rotation, the tensor $\sigma_{a b}$ the anisotropic expansion and the scalar $\theta$ the isotropic expansion of $\perp \delta x^{a}$. The dynamic variables of the spacetime are defined by the $1+3$ decomposition of the Einstein tensor $G_{a b}$ as follows 33

$$
G_{a b}=\rho u_{a} u_{b}+2 q_{(a} u_{b)}+p h_{a b}+\pi_{a b}
$$

where $\rho=G_{a b} u^{a} u^{b}$ is the energy-mass density of the fluid, $p=\frac{1}{3} h^{a b} G_{a b}$ is the isotropic pressure, $q^{a}=-h^{a c} G_{c d} u^{d}$ is the heat flux tensor and $\pi_{a b}=\left(h_{a}^{c} h_{b}^{d}-\frac{1}{3} h^{c d} h_{a b}\right) G_{c d}$ is the traceless anisotropic tensor (measures the anisotropy of the fluid).

Applying the above for the comoving observers in Bianchi III spacetime (22) we compute that the kinematic quantities $\omega^{2}=0, \alpha^{a}=0$ while

$$
\theta=\frac{e^{-\frac{m \lambda}{2} \tau}}{A}\left[3 \frac{d(\ln A)}{d \tau}+\frac{m(2 \lambda-1)}{2}\right]
$$

and

$$
\sigma^{2}=\frac{m^{2}\left(\lambda^{2}-\lambda+1\right)}{12} \frac{e^{-m \lambda \tau}}{A^{2}} .
$$

Similarly for the dynamic quantities we find that the (non-zero) components for the cosmological fluid defined by the Bianchi III spacetime (22) are

$$
\begin{gathered}
\rho=\frac{e^{-m \lambda \tau}}{4 A^{2}}\left[4 \frac{d(\ln A)}{d \tau}\left(3 \frac{d(\ln A)}{d \tau}+m(2 \lambda-1)\right)+m^{2} \lambda(\lambda-1)-4\right] \\
p=\frac{e^{-m \lambda \tau}}{A^{2}}\left[-\frac{2}{A} \frac{d^{2} A}{d \tau^{2}}+\frac{d(\ln A)}{d \tau}\left(\frac{d(\ln A)}{d \tau}+\frac{m}{3}(2-\lambda)\right)-\frac{m^{2}}{12}(\lambda-1)(\lambda-2)+\frac{1}{3}\right] \\
q^{a}=\left(0, \frac{m \lambda}{2} \frac{e^{-\frac{3 m \lambda}{2} \tau}}{A^{3}}, 0,0\right) \\
\pi_{x x}=\frac{m(\lambda+1)}{3} \frac{d(\ln A)}{d \tau}+\frac{m^{2}\left(\lambda^{2}-1\right)}{12}-\frac{1}{3} \\
\pi_{y y}=e^{-m \tau}\left[\frac{m(\lambda-2)}{3} \frac{d(\ln A)}{d \tau}+\frac{m^{2}}{12}(\lambda-1)(\lambda-2)+\frac{2}{3}\right]
\end{gathered}
$$

and

$$
\pi_{z z}=-e^{-m \lambda \tau-2 x}\left[\frac{m(2 \lambda-1)}{3} \frac{d(\ln A)}{d \tau}+\frac{m^{2}}{12}(\lambda-1)(2 \lambda-1)+\frac{1}{3}\right] .
$$

In the case of $A^{2}(\tau)=e^{m(\kappa-\lambda) \tau}$, where the CKV $\mathbf{L}_{1}$ becomes a $\mathrm{HV}$, the above non-zero quantities are simplified as follows

$$
\begin{gathered}
\theta=\frac{m(3 \kappa-\lambda-1)}{2} e^{-\frac{m \kappa}{2} \tau} \\
\sigma^{2}=\frac{m^{2}\left(\lambda^{2}-\lambda+1\right)}{12} e^{-m \kappa \tau} \\
\rho=\rho_{0}(m, \kappa, \lambda) e^{-m \kappa \tau}, p=p_{0}(m, \kappa, \lambda) e^{-m \kappa \tau} \\
q^{a}=\left(0, \frac{m \lambda}{2} e^{-\frac{3 m \kappa}{2} \tau}, 0,0\right) \\
\pi_{x x}=\pi_{x x 0}(m, \kappa, \lambda), \pi_{y y}=\pi_{y y 0}(m, \kappa, \lambda) e^{-m \tau}, \pi_{z z}=\pi_{z z 0}(m, \kappa, \lambda) e^{-m \lambda \tau-2 x} .
\end{gathered}
$$

From the latter expressions we infer that for large $\tau$ and $m \kappa>0$ all the kinematical quantities, the mass density, the isotropic pressure and the heat flux vector vanish. If, in addition, $\pi_{x x 0}(m, \kappa, \lambda)=0, m>0$ and $\lambda>0$, then for large $\tau$ the fluid source vanishes and the solution describes an isotropic empty spacetime. 


\subsection{Bianchi V cosmological fluid}

We consider the extended Bianchi V spacetime of proposition 2

$$
d s_{(V)}^{2}=-A^{2}(\tau) e^{m \lambda \tau} d \tau^{2}+A^{2}(\tau) e^{m \lambda \tau} d x^{2}+A^{2}(\tau) e^{m(\lambda-1) \tau} e^{2 x} d y^{2}+A^{2}(\tau) e^{2 x} d z^{2}
$$

and repeat the calculations for the comoving observers. We find that the kinematic quantities are exactly the same with those of the Bianchi III spacetime while the dynamic (non-zero) dynamic variables of the cosmological fluid are

$$
\begin{gathered}
\rho=\frac{e^{-m \lambda \tau}}{4 A^{2}}\left[4 \frac{d(\ln A)}{d \tau}\left(3 \frac{d(\ln A)}{d \tau}+m(2 \lambda-1)\right)+m^{2} \lambda(\lambda-1)-12\right] \\
p=\frac{e^{-m \lambda \tau}}{A^{2}}\left[-\frac{2}{A} \frac{d^{2} A}{d \tau^{2}}+\frac{d(\ln A)}{d \tau}\left(\frac{d(\ln A)}{d \tau}+\frac{m}{3}(2-\lambda)\right)-\frac{m^{2}}{12}(\lambda-1)(\lambda-2)+1\right] \\
q^{a}=\left(0,-\frac{m(\lambda+1)}{2} \frac{e^{-\frac{3 m \lambda}{2}}}{A^{3}}, 0,0\right) \\
\pi_{x x}=\frac{m(\lambda+1)}{3} \frac{d(\ln A)}{d \tau}+\frac{m^{2}\left(\lambda^{2}-1\right)}{12} \\
\pi_{y y}=e^{-m \tau+2 x}\left[\frac{m(\lambda-2)}{3} \frac{d(\ln A)}{d \tau}+\frac{m^{2}}{12}(\lambda-1)(\lambda-2)\right]
\end{gathered}
$$

and

$$
\pi_{z z}=-e^{-m \lambda \tau+2 x}\left[\frac{m(2 \lambda-1)}{3} \frac{d(\ln A)}{d \tau}+\frac{m^{2}}{12}(\lambda-1)(2 \lambda-1)\right] .
$$

In the case $\mathbf{L}_{1}$ is a $\mathrm{HV}$ we deduce the same conclusions with the Bianchi III case of section 5.1 .

\section{Lie point symmetries of the wave equation}

Collineations of spacetimes can be used to construct symmetries and conservation laws for some differential equations defined in curved spacetimes. In [34] it has been shown that there exists a unique connection between the Noether symmetries for the geodesic Lagrangian of a given Riemannian space and the elements of the admitted homothetic algebra. Similar results have been shown for other partial differential equations of special interest [35, 36].

In this work we consider the wave equation

$$
\frac{1}{\sqrt{-g}} \frac{\partial}{\partial x^{\mu}}\left(\sqrt{-g} g^{\mu \nu} \frac{\partial}{\partial x^{\nu}}\right) u\left(x^{\lambda}\right)=0
$$

in the Bianchi III spacetime (22) and in the Bianchi V spacetime (27) and determine its Lie symmetries. By following the generic results of [36], we find that the admitted Lie point symmetries of the wave equation in the Bianchi III spacetime (22) are the $3 \mathrm{KVs}$, the vector field $Y_{u}=u \partial_{u}$, and the infinitely many vectors $Y_{\infty}=b\left(x^{\mu}\right) \partial_{u}$ where $b\left(x^{\mu}\right)$ is a solution of the original equation (50). The latter symmetry vector fields exist because equation (50) is a linear partial differential equation.

For a higher dimensional conformal algebra, equation (50) admits extra Lie point symmetries. Indeed, from our analysis and for the case where the Bianchi III and Bianchi V spacetimes admit a proper HV the wave equation becomes

$$
\left(-u_{t t}+u_{x x}+u_{y y}+e^{m \lambda t+2 x} u_{z z}\right)+\frac{m}{2}(\lambda-2 \kappa+1) u_{t}-u_{x}=0
$$

or

$$
\left(-u_{t t}+u_{x x}+e^{m t-2 x} u_{y y}+e^{m \lambda t-2 x} u_{z z}\right)+\frac{m}{2}(\lambda+2 \kappa-1) u_{t}+4 u_{x}=0 .
$$

Then we find that equation (51) admits the generic Lie point symmetry vector

$$
Y_{I I I}=\left(a_{1} \frac{2}{m}\right) \partial_{t}+a_{2} \partial_{x}+\left(a_{1} y+a_{3}\right) \partial_{y}+\left(a_{1} \lambda z+a_{2} z+a_{4}\right) \partial_{z}+\left(a_{u} u+a_{\infty} b(t, x, y, z)\right) \partial_{u}
$$


while equation (52) is invariant under the one parameter point transformation with generator

$$
Y_{V}=\left(a_{1} \frac{2}{m}\right) \partial_{t}+a_{2} \partial_{x}+\left(a_{1} y-a_{1} y+a_{3}\right) \partial_{y}+\left(a_{1} \lambda z-a_{2} z+a_{4}\right) \partial_{z}+\left(a_{u} u+a_{\infty} b(t, x, y, z)\right) \partial_{u}
$$

The latter symmetry vectors can be applied to construct conservation laws or similarity solutions for the wave equation. However, such an analysis is beyond the scope of the present work.

\section{Conclusion}

In this paper we have shown that there is only one type of Bianchi III and Bianchi V spacetime given respectively in (22) and (27) which admit a single proper CKV. Furthermore, two more spacetimes are found which admit a HV. In order to arrive at this result we applied an algorithm which relates the CKVs of decomposable spacetimes with the collineations of the non-decomposable subspace. The kinematics of the fluid of the comoving observers in all these four spacetimes is not accelerating and rotating and has only expansion and shear a result compatible with the anisotropy of the Bianchi spacetimes. Concerning the dynamics it has been shown that the fluid of these observers is heat conducting and anisotropic, that is it is a general fluid. Finally we have used the conformal vectors we found in each case in order to determine the generators of the Lie symmetries of the wave equation in the Bianchi III spacetime (22) and in the Bianchi V spacetime (27).

\section{Acknowledgement}

AP thanks the University of Athens for the hospitality provided while this work was carried out.

\section{References}

[1] R. Maartens, D. P. Mason and M. Tsamparlis, J. Math. Phys. 27, 2987 (1986)

[2] A. A. Coley and B. O. J. Tupper, J. Math. Phys. 33, 1754 (1992)

[3] A. A. Coley and B. O. J. Tupper, Class. Quantum Grav. 11, 2553-2574 (1994)

[4] R. Maartens, S. D. Maharaj and B. O. J. Tupper, Class. Quantum Grav. 12, 2577-2586 (1995)

[5] M. Tsamparlis, A. Mitsopoulos and A. Paliathanasis, A. Gen. Relativ. Gravit. 51: 6 (2019)

[6] A.A. Coley and B.O.J. Tupper, Class.Quantum Grav 7, 1961 (1990)

[7] A.A. Coley and B.O.J. Tupper, Class.Quantum Grav 7, 2195 (1990)

[8] L. Herrera, J. Ponce de Leon J.Math.Phys 26, 2332 (1985)

[9] L. Herrera, J. Ponce de Leon J.Math.Phys 26,2018 (1985)

[10] C. Frances, Geom Dedicata 158, 35-59 (2012)

[11] P. Joharinad and B. Bidabad, Diff Geom and its App 31, 33 (2013)

[12] R. Geroch, J. Math. Phys. 12, 918 (1971)

[13] R. Geroch, J. Math. Phys. 13, 394 (1972)

[14] M. Tsamparlis and A. Paliathanasis, Gen. Relativ. Gravit. 42, 2957 (2010)

[15] G.H. Katzin and J. Levine, J. Math. Phys. 9, 8 (1968)

[16] G.H. Katzin and J. Levine, J. Math. Phys. 22, 1878 (1981)

[17] A.V. Aminova, Sbornik Math. 186, 1711 (1995) 
[18] A.V. Aminova, Tensor N.S., 65, 62 (2000)

[19] M. Tsamparlis and A. Paliathanasis, Nonlinear Dynamics 62, 203 (2010)

[20] M. Tsamparlis and A. Paliathanasis, Gen. Relativ. Gravit. 42, 2957 (2010)

[21] Y. Bozhkov and I.L. Freire, J. Diff. Eq. 249, 872 (2010)

[22] A. Paliathanasis and M. Tsamparlis, J. Geom. Phys. 107, 45 (2016)

[23] A. Paliathanasis and M. Tsamparlis, J. Geom. Phys. 124, 165 (2018)

[24] M. Tsamparlis, D. Nikolopoulos and P.S. Apostolopoulos, Class. Quant. Grav. 15, 2909 (1998)

[25] P. S. Apostolopoulos and M. Tsamparlis, Tensor (Japan) 61, 260, (1999)

[26] A. Karagiorgos, T. Pailas, N. Dimakis, P.A. Terzis and T. Christodoulakis, JCAP 03, 030 (2018)

[27] A. Krasiński, Inhomogeneous Cosmological Models, Cambridge University Press, New York (1997)

[28] M. Tsamparlis, A. Paliathanasis and L. Karpathopoulos, Gen. Rel. Gravit. 47, 15 (2015)

[29] H. Stephani, D. Kramer, M. Maccallum, C. Hoenselaers and E. Herlt, "Exact Solutions to Einstein's Field Equations (2nd edition)", Cambridge University Press, (2009)

[30] R. M. Wald, "General Relativity", Chicago University Press, (1984)

[31] M. P. Ryan and L. C. Shepley "Homogeneous Relativistic Cosmologies", Princeton University Press, (1975)

[32] P. S. Apostolopoulos and M. Tsamparlis, Class. Quant. Grav. 18, 3775 (2001)

[33] G.F.R. Ellis and H. van Elst and, "Cosmological models", Cargèse Lectures (1998)

[34] M. Tsamparlis and A. Paliathanasis, Gen. Relativ. Gravit. 43, 1861-1881 (2011)

[35] A. Paliathanasis and M. Tsamparlis, J. Geom. Phys. 62, 2443 (2012)

[36] A. Paliathanasis and M. Tsamparlis, Int. J. Geom. Meth. Mod. Phys. 11, 1450037 (2014) 